ARTICLE

DOI: $10.1038 / s 41467-017-02562-5$

\title{
A biosensor-based framework to measure latent proteostasis capacity
}

\author{
Rebecca J. Wood ${ }^{1}$, Angelique R. Ormsby ${ }^{1}$, Mona Radwan', Dezerae Cox¹, Abhishek Sharma², Tobias Vöpel (10 2, \\ Simon Ebbinghaus ${ }^{2}$, Mikael Oliveberg ${ }^{3}$, Gavin E. Reid ${ }^{1,4}$, Alex Dickson ${ }^{5,6}$ \& Danny M. Hatters (i) ${ }^{1}$
}

The pool of quality control proteins (QC) that maintains protein-folding homeostasis (proteostasis) is dynamic but can become depleted in human disease. A challenge has been in quantitatively defining the depth of the QC pool. With a new biosensor, flow cytometrybased methods and mathematical modeling we measure the QC capacity to act as holdases and suppress biosensor aggregation. The biosensor system comprises a series of barnase kernels with differing folding stability that engage primarily with HSP7O and HSP9O family proteins. Conditions of proteostasis stimulation and stress alter QC holdase activity and aggregation rates. The method reveals the HSP7O chaperone cycle to be rate limited by HSP70 holdase activity under normal conditions, but this is overcome by increasing levels of the BAG1 nucleotide exchange factor to HSPA1A or activation of the heat shock gene cluster by HSF1 overexpression. This scheme opens new paths for biosensors of disease and proteostasis systems.

\footnotetext{
${ }^{1}$ Department of Biochemistry and Molecular Biology, Bio21 Molecular Science and Biotechnology Institute, University of Melbourne, Parkville, VIC 3010 , Australia. ${ }^{2}$ Department of Physical Chemistry II, Ruhr-University Bochum, Universitaetsstraße 150, 44780 Bochum, Germany. ${ }^{3}$ Department of Biochemistry and Biophysics, Arrhenius Laboratories of Natural Sciences, Stockholm University, 10691 Stockholm, Sweden. ${ }^{4}$ School of Chemistry, University of Melbourne, Parkville, VIC 3010, Australia. ${ }^{5}$ Department of Biochemistry \& Molecular Biology Michigan State University, East Lansing, MI 48824, USA. ${ }^{6}$ Department of Computational Mathematics, Science and Engineering Michigan State University, East Lansing, MI 48824, USA. Correspondence and requests for materials should be addressed to D.M.H. (email: dhatters@unimelb.edu.au)
} 
P rotein-folding homeostasis (proteostasis) in humans is controlled by a quality control (QC) network of about 800 proteins ${ }^{1}$. Key cogs of the QC network are the chaperones, such as HSP70 and HSP90 family members, which monitor the foldedness of proteins almost from the moment they emerge from the ribosome and thereon throughout their lifespan. While the QC network can dynamically respond to stresses to maintain proteostasis, it can also become depleted in protein misfolding diseases $^{1-3}$. A challenge has been to define the buffering depth of the QC network in managing proteostasis and to track how it changes when stimulated or challenged.

To understand baseline proteostasis buffering capacity requires the development of new quantitative approaches. Prior schemes have used aggregation of ectopically expressed conformationally destabilized (i.e., metastable) proteins as flags for when proteostasis had declined ${ }^{4-6}$. These schemes operated on the principle that QC systems actively suppress the aggregation rates of metastable "bait" proteins and hence when proteostasis was depleted cells lost the capacity to suppress aggregation. Others have also examined the in-cell folding rates of a test protein through rapid temperature jumps to follow rates for reestablishment of equilibrium ${ }^{7}$. However, these approaches lack a quantitative capacity to understanding proteostasis; namely through the inability to define the effectiveness of QC systems to engage with the bait proteins.

Our motivation was to develop a new biosensor system modeled on these prior schemes-but with a substantially improved quantitative capacity. Here, we describe a biosensor system based on a series of metastable bait proteins that report on foldedness and aggregation state by fluorescence resonance energy transfer (FRET). We show that we can measure the engagement of QC (in terms of the net holdase activity from many individual components) to the unfolded state of the biosensor and concomitant influences on the biosensor aggregation. We describe a new mathematical framework that can extract quantitative information from the holdase activity of QC as well as ability to suppress aggregation. These approaches provide insight to the depth of the pool of QC resources that regulate proteostasis.

\section{Results}

Barnase as a sensing kernel for a new tunable biosensor. The prior biosensor schemes using metastable proteins typically display complex folding mechanisms, which make good substrates for QC but pose great challenges in mechanistically quantifying the effects in terms of the thermodynamics of the system ${ }^{4-6}$. We hence chose a bait protein through which we could more deeply examine the thermodynamics of protein folding and aggregation in the context of QC engagement. Barnase (in a catalytically inactive form (H102A mutant $\left.{ }^{8}\right)$-defined hereon as wild-type based on established nomenclature), was chosen as our bait because it can be predictably tuned to different free energies of folding $\left(\Delta G_{F}\right)$ by mutation ${ }^{9-11}$. Knowledge of, and the capacity to predictably alter $\Delta G_{F}$, provides a strategy to alter the dynamic range of foldedness and hence provide a handle for more control over defining the extent to which proteostasis alters the folded state of barnase away from thermodynamic equilibrium and basal aggregation levels (Fig. 1).

We predicted that a FRET strategy-flanking the barnase moiety with two fluorescent proteins-could be used to monitor barnase conformations; including chaperone-unfolded client complex (Fig. 1). To make the biosensor, we first screened a mini-library of circularly permuted variants of monomeric teal fluorescent protein 1 (mTFP1) and Venus to identify the optimal combination for tracking barnase foldedness (Supplementary Fig. 1). Upon determining the best combination (mTFP1 cp175-

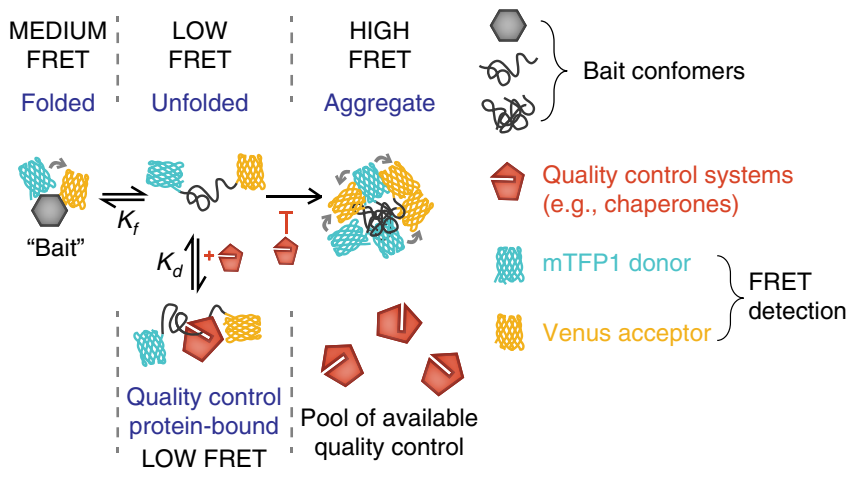

Fig. 1 Design strategy for probing proteostasis efficiency. Scheme of how the bait biosensor module measures collective cellular chaperone engagement by binding to unfolded barnase and in preventing aggregation. Medium FRET and low FRET: binding of quality control machinery to barnase bait pulls the equilibrium away from folded barnase, reducing the FRET signal in the soluble pool. High FRET: quality control systems reduce aggregate accumulation, thereby reducing the number of cells with highFRET

barnase-Venus cp173), we made 15 mutants predicted to span a range of equilibrium constants of folding $\left(K_{f}\right)$, corresponding to $\Delta G_{F}$ values between $-25 \mathrm{~kJ} / \mathrm{mol}$ (most stable) to $1 \mathrm{~kJ} / \mathrm{mol}$ (least stable $)^{10,11}$. Denaturation curves of these constructs in purified form or directly in mammalian lysates yielded $\Delta G_{F}$ values that correlated with the previously recorded or predicted $\Delta G_{F}$ values $^{10,11}$, demonstrating that the FRET scheme authentically reports on barnase folding equilibrium (Fig. 2a, b; Supplementary Fig. 2a, b; Supplementary Table 1). Furthermore, assessment of FRET in cells expressing the mutant biosensor revealed the cells containing extensively aggregated biosensor have higher FRET signal than cells lacking aggregates, suggesting that aggregation can be detected as a higher FRET state than the folded and unfolded states (Fig. 2c). Hence, these data confirm the capacity to follow the three FRET states as depicted in Fig. 1: high-FRET (aggregated), medium-FRET (folded), and low-FRET (unfolded).

Next we assessed whether barnase was indeed an appropriate client for sampling mammalian QC. Binding partners to the biosensor were measured by quantitative proteomics on immunoprecipitates of a barnase biosensor tuned to be folded (wildtype ${ }^{\star}$ ) vs. a variant tuned to be substantially unfolded (I25A, I96G). A variety of heat shock family proteins bound abundantly to both forms of the biosensor based on the Exponentially Modified Protein Abundance Index (emPAI) score ${ }^{12}$. Four of the five most enriched binding partners to the more unfolded biosensor (with a $p<0.01$, Student's $t$-test) were HSP70 family members (3.9-fold more HSPA1B, 1.7-fold more HSPA8) and HSP90 family members (1.9-fold more HS90AB1 and HS90AA1) (Supplementary Table 2). Furthermore, these four chaperones were the most abundant proteins in the immunoprecipitants based on the emPAI score ${ }^{12}$. Hence, we concluded that the basic barnase module is a suitable probe for some of the major chaperones of the QC network.

Two quantitative measures of QC engagement. To calculate the extent of QC engagement (which we hereon refer to in terms of holdase activity) we needed to devise a strategy to distinguish the conformers of barnase as depicted in Fig. la in intact live cells. To do this, we used flow cytometry to measure donor and FRET (i.e., sensitized emission) channels. For the wild-type* barnase biosensor, we saw a strict linear relationship between donor and FRET emission (Fig. 3a). Because the slope of this dependence is 
a

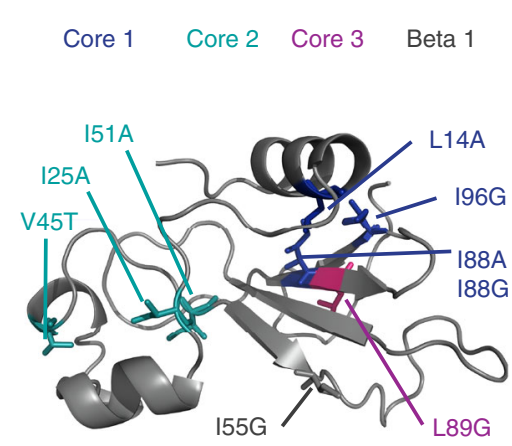

b

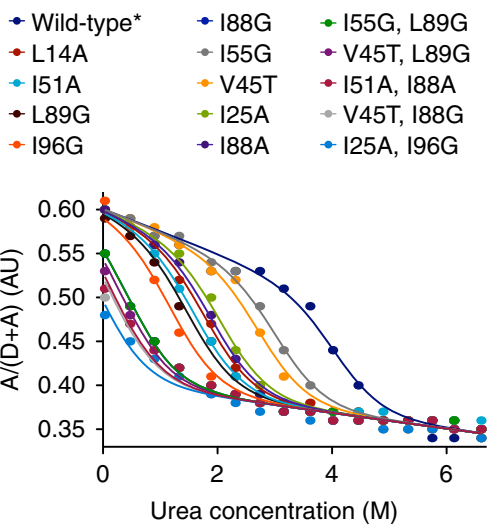

C

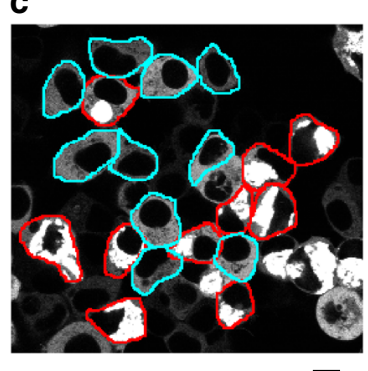

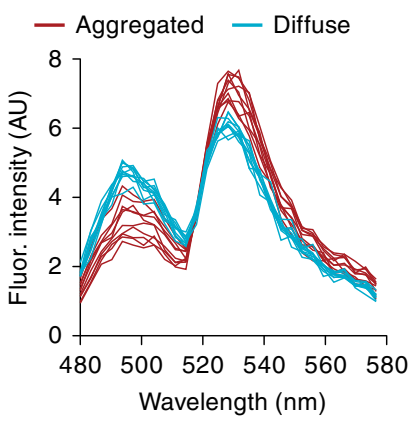

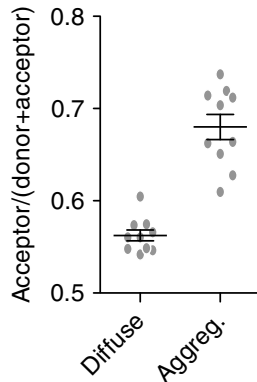

Fig. 2 FRET reports on barnase foldedness and aggregation. a Bait protein barnase structure is shown (PDB ID 1A2P) with the location of destabilizing mutations used to tune $K_{f}$ (and thus $\Delta G_{F}$ ). b Urea denaturation curves, assayed by FRET, are shown of barnase constructs expressed in mammalian lysate fitted to a two-state unfolding model (one representative replicate per mutant of $n=3$ ). c A confocal micrograph image of representative cells expressing a destabilized barnase variant (I25A, 196G) in HEK293T cells. Scale bar=10 $\mu \mathrm{m}$. The wild-type barnase variant does not form visible aggregates. The middle graph shows fluorescence spectra (excitation $405 \mathrm{~nm}$ ) for cells with only diffuse biosensor vs. cells with visible aggregates. The right graph shows a proxy measure for FRET of these cells (means \pm SEM)

proportional to FRET efficiency (i.e., a higher FRET will result in a greater slope), its linearity indicated that all cells belong to a single FRET population with similar fraction of folded barnase, which we anticipated would be close to $100 \%$ based on the $\Delta G_{F}$ value of $-25 \mathrm{~kJ} / \mathrm{mol}$. By contrast, cells expressing destabilized barnase mutants, such as the V45T, L89G double mutant, segregated into two populations with different FRET slopes (Fig. 3a). Sorted cells from the Lower-slope population were enriched with unaggregated barnase, whereas those in the Upper-slope were enriched with visibly aggregated barnase, accounting for the increased FRET (Fig. 3b). These data suggested that the gradient of the Lower-slope population provided a measure of the balance of folded and unfolded barnase states in the absence of aggregation (Fig. 3c). This conclusion was supported by the Lowerslope gradients of each mutant correlating tightly with the fraction folded expected from the measured $\Delta G_{F}$ (Fig. 3d-the slopes for select data in the figures are presented in Supplementary Data 1; Supplementary Note 1 for more discussion on this point).

To mechanistically explain QC engagement from these data, we considered a simple model for the cell population lacking aggregates (Lower-slope population). In these cells our model assumed three barnase states dominate the molecular pool: folded, free unfolded and unfolded bound to chaperones (and other QC proteins hereon referred to as chaperones for simplicity $)^{13}$. We assumed that the free unfolded state and unfolded-chaperone bound states have similar low FRET values on the basis that HSP70 family proteins (as well as other chaperones) can act as a holdase to unfolded client ${ }^{14}$. We postulated that when the cell has a higher "holdase" capacity there will be a greater pool of latent chaperone $(C)$, available to bind unfolded barnase and increasingly partition unfolded barnase from the thermodynamic equilibrium of folding (Fig. 4a). Barnase folding and unfolding rates are typically on the scale of milliseconds to seconds ${ }^{15}$. Assuming that synthesis, degradation and aggregation rates are slower than this-synthesis occurs in mammals on the scale of at least $10 \mathrm{~s}$ of seconds per protein ${ }^{16}$ and degradation occurs on the rate of minutes to hours ${ }^{17}$ - the ratio between native and free unfolded barnase $\left(K_{f}\right)$ will remain the same at equilibrium, but both states will decrease in concentration at higher proteostasis capacity.

To test this model, we overexpressed human chaperones HSPA1A and DNAJB1 (family members of HSP70 and HSP40 respectively). Immunoprecipitation experiments of a biosensor variant tuned to be substantially unfolded (I25A, I96G) verified this treatment produced a greater interaction of HSPA1A with the biosensor as anticipated from elevated holdase activity (Supplementary Fig. 3a-c). And in accordance with the model, this treatment also decreased the Lower-slope values compared to a negative control biosensor lacking the barnase kernel, consistent with a greater holdase activity to the unfolded biosensor (Fig. 4b).

Next we sought to use the model to define the extent of the pool of QC resources capable of holdase activity, defined here as the latent chaperone concentration $(C)$. Changes in $C$ between a control condition and treatment $(\Delta C)$ can be determined with knowledge of a binding affinity constant for the average interactions of all chaperones in the cell with barnase $\left(K_{d}\right)$, the fraction folded of a given barnase mutant under control $\left(f_{c}\right)$ and 
a
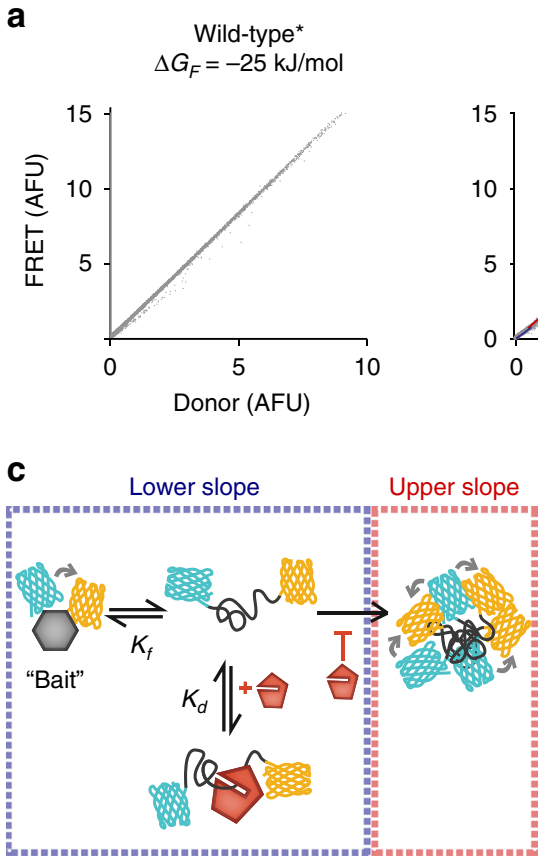

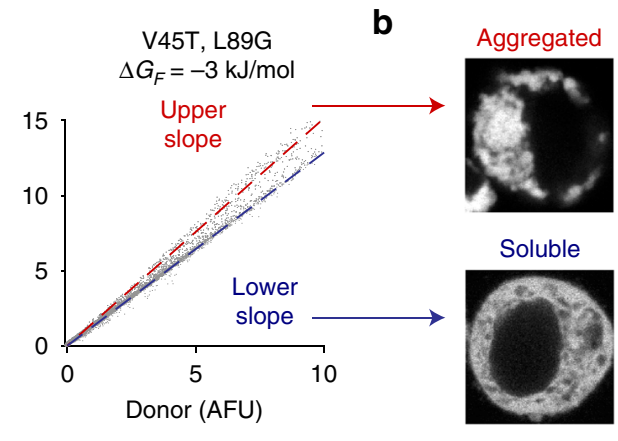

d

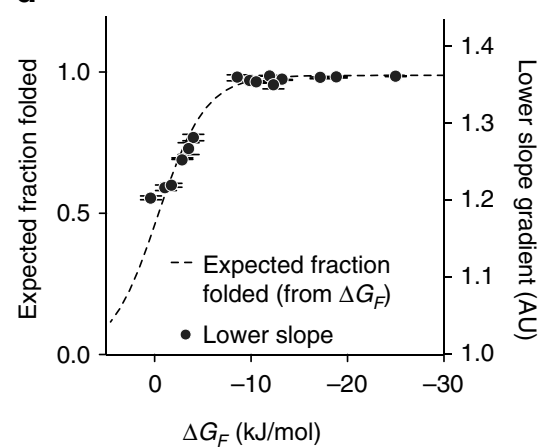

Fig. 3 Barnase foldedness and aggregation can be readily assessed by flow cytometry. a Flow cytometry analysis to determine FRET. Data points indicate fluorescence signatures of individual cells for donor and FRET channels. $\mathbf{b}$ Representative confocal images of cells recovered by cell sorting (scale bar=10 $\mu \mathrm{m}$; "Soluble" image scaled at $4 \times$ brightness vs. "Aggregated"). c Conceptual framework for how flow cytometry data reports on foldedness vs. aggregation d The Lower-slope gradients of barnase mutants measured by flow cytometry (right axis) follows the expected relationship between stability ( $\Delta G_{F}$ ) and fraction folded (left axis, scaled to fit). Each data point reflects one barnase mutant (mean \pm SD; three replicates). Note that the AFU or AU scales, while arbitrary, cannot be compared between different panels and figures due to changes in instrument calibration settings

treatment conditions $\left(f_{t}\right)$, folding equilibrium constant $K_{f}$, and the barnase concentration $(B)$

$$
\Delta C=\frac{-K_{d} K_{f}\left(f_{t}-f_{c}\right)}{f_{t} f_{c}}-B\left(f_{t}-f_{c}\right)\left(1+\frac{1}{K_{f}}\right)
$$

Analysis of the Lower-slope data for the set of barnase mutants upon the DNAJB1 and HSPA1A over-expression enabled a calculation of $\Delta C$ (Fig. 4c) and hence provided a useful scalar measure of QC holdase activity. Details of the analysis are explained in Supplementary Note 2 (for derivation) and Supplementary Note 3 (for application of Equation 1 to our data).

To provide a quantitative measure of aggregation, we investigated the Upper-slope FRET population and the proportion of cells that fell into this population (Fig. 5a). As anticipated, the proportion of cells containing barnase aggregates increased in correlation with biosensors tuned to more positive $\Delta G_{F}$ values and also to higher protein levels in each cell (Fig. 5b). Overexpression of DNAJB1 and HSPA1A increased the concentration-threshold of aggregation for two of the barnase variants (I51A and V54T, I88G), consistent with chaperonemediated suppression of aggregation (Fig. 5c). This data indicated that changes in aggregation propensity could be measured by the barnase concentration at which $50 \%$ of the cells contained aggregates at a particular time point of expression $\left(A_{50 \%}\right)$ (Fig. $5 \mathrm{c}$ ). When all the mutants were considered, it was apparent that the $A_{50 \%}$ correlated linearly to $\Delta G_{F}$ (Fig. $5 \mathrm{~d}$ ). The DNAJB1 and HSPA1A overexpression treatment offset $A_{50 \%}$ by a comparable amount for all barnase mutants (Fig. 5d). These results collectively indicated that the change in proteostasis efficiency can be measured as a single scalar parameter by the translational offset due to the treatment of interest $\left(\Delta A_{50 \%}\right)$ (Fig. $5 \mathrm{e}$; further discussion of the $A_{50 \%}$ analysis is provided in Supplementary Note 1).

Using the biosensor to probe how QC manages proteostasis. Next we assessed whether we could use the biosensor to measure changes in QC engagement upon stress of proteostasis. First we inhibited HSP90 with novobiocin, which impairs HSP90 activity without activating the heat shock response ${ }^{18,19}$. We predicted this treatment would lead to negative $\Delta C$ and $\Delta A_{50 \%}$ values, which was the case (Figs $4 \mathrm{~d}$ and $5 \mathrm{e}$ ). Furthermore, the activity of novobiocin could be extracted with this method in terms of a dose response curve, providing an $\mathrm{IC}_{50}$ in reasonable accordance with its known value $\left(1.8 \mathrm{mM}\right.$ (our data) c.f. $\approx 700 \mu \mathrm{M}$ (literature) ${ }^{18}$; Supplementary Fig. 4). As a control, we overexpressed HSP90 family member HSP90AA1, which led to the anticipated reverse response with respect to suppression of aggregation although the change in holdase activity was not significant (Fig. 6a). As a second test for stress, we depleted ATP levels using a glycolysis inhibitor (2-deoxy-d-glucose) in combination with an inhibitor of oxidative phosphorylation (valinomycin) ${ }^{20,21}$ (Supplementary Fig. 5). Under such conditions, we predicted that chaperone networks would be impaired from functioning, and hence lead to a net loss of engagement with client. This appeared to be the case, with a negative $\Delta C$ (Fig. $4 \mathrm{~d})$ resulting as well as a negative $\Delta A_{50 \%}$, (Fig. 5e).

Next we explored the capacity of the biosensor system to gain insight into the mechanics of QC systems that regulate proteostasis. First, we applied a selective inhibitor of HSP70 ATPase activity VER-155008, which binds competitively to the ATP binding pocket of HSP70 family proteins ${ }^{22}$. While this inhibitor does not prevent client binding in vitro ${ }^{23}$, it 

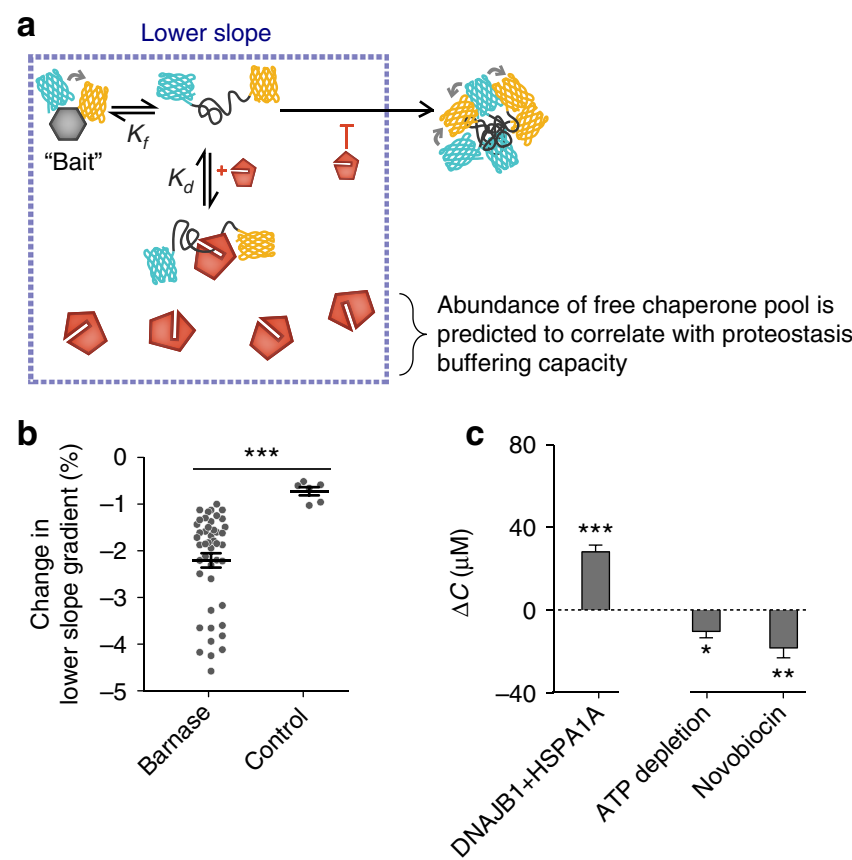

Fig. 4 Quantifying proteostasis by changes in latent chaperone concentration available for holdase activity $(\Delta C)$. a Conceptual framework for how chaperone levels affect barnase foldedness. b Changes in Lowerslope gradients of the barnase mutants (data points show individual replicates of each mutant) vs. a FRET-positive negative control (mTFP1 cp175-Venus cp173 fusion lacking the barnase kernel; data points show individual replicates) when co-expressed with HSP4O and HSP7O chaperones (DNAJB1 and HSPA1A respectively) relative to baseline conditions (Y66L EGFP co-expression). Means \pm SEM shown. c Effect of toggling proteostasis on changes in available chaperone capacity $(\Delta C)$. Plots show means \pm SEM of the 12 barnase mutants when coexpressed with DNAJB1 and HSPA1A (compared to Y66L EGFP control; left panel), or treated with proteostasis-modulating drugs (compared to untreated control). Wilcoxon signed rank tests results coded as ${ }^{\star \star \star} p<0.001,{ }^{\star \star} p<$ $0.01,{ }^{\star} p<0.05$

did lead to an increase in the Lower-slope values and decrease in $A_{50 \%}$ consistent with an overall reduction in the pool of chaperone supply with dynamic capacity to act as a holdase and suppress aggregation (Supplementary Fig. 6).

Next we probed HSP70-HSP40 holdase activity in greater context of nucleotide exchange factors (NEFs), which catalyze the disengagement of client bound to HSP40-HSP70 to complete the chaperone cycle ${ }^{24-26}$. As described above (Fig. 4c, d), overexpression of HSPA1 and DNAJB1 increased the net unfolded pool of the biosensor through a gain in holdase activity. This suggested that overloading a cell with HSPA1 and DNAJB1 by their selective overexpression created a bottleneck in QC capacity to complete the chaperone cycle. To test this possibility, we coexpressed BAG1, the NEF co-factor to HSPA1, with HSPA1 and DNAJB1 (Fig. 6b). This treatment significantly increased foldedness of a biosensor tuned to be moderately unfolded (L89G) compared to when HSPA1 and DNAJB1 were overexpressed without BAG1. This is hence consistent with a decrease in HSP70 holdase activity as anticipated by a greater NEF activity to facilitate release of client bound to HSPA1 and DNAJB1. Assessment of aggregation by $\Delta A_{50 \%}$ analysis revealed the NEF to not provide any further enhancement to proteostasis than HSPA1 and DNAJB1 overexpression without BAG1, suggesting that the holdase activity may be sufficiently effective to mitigate inappropriate aggregation (Fig. 6b).
An interesting result from this experiment was that the NEFHSP40-HSP70 overexpression increased foldedness of the biosensor to a greater extent than what was observed under baseline conditions (Fig. 6b). This suggested that the HSP70 chaperone cycle did not operate at maximum potential under normal conditions whereby NEF activity was the rate limiting step. To further probe this possibility, we overexpressed HSF1, which induces expression of the heat shock response genes (Fig. 6a). This treatment significantly increased foldedness of the L89G variant biosensor (and also suppressed its aggregation) beyond baseline conditions (Fig. 6a). Hence, this supported the conclusion that HSP70 chaperone activity is ratelimited under normal conditions by NEF activity and that this can be overcome by a coordinated upswing in cellular QC resources driven through the heat shock response gene elements.

\section{Discussion}

We describe here a strategy for quantifying the holdase activity of the QC network as an indicator of proteostasis health. Using barnase as a bait protein to the QC we were able to define the extent to which the QC system altered the folding equilibrium and aggregation and also validate how manipulation of QC machinery alters the biosensor readout in a predictable way. The key objective of the biosensor was to provide a simple numerical metric of "holdase" activity and capacity to suppress aggregation. As such, this system provides a quantitative measure of the health of proteostasis. However, as indicated by the studies with selective chaperone overexpression and proteomics, the biosensor also has capacity for nuanced analysis of the specific changes in chaperones that interact with the biosensor under different conditions. One area of potential application is in the study of selectivity in how different HSP70 proteins respond to client under different stresses. Prior work has suggested HSP70 family members display selectivity in function: some are better at refolding misfolded proteins and others at preventing aggregation ${ }^{27}$. Others seem to be involved in protein disaggregation ${ }^{28}$. The biosensor platform described here provides a manner to explore the dual role of holdase activity and aggregation of individual HSP70 proteins (e.g., by their selective knock out) in an intact proteostasis system. An interesting finding was how the HSP70 chaperone cycle appeared to not be operating a maximal capacity under baseline conditions. This may provide a mechanism for HSP70 to accumulate client as part of HSP70-mediated client triage to other nodes of the QC system, such as degradation ${ }^{29}$. It may also provide QC systems an instant buffering strategy after an acute stress during the time period for stress responses to synthesize additional QC resources.

Collectively, the new biosensor system and methods described here offer promise to probe mechanisms intersecting proteostasis and disease. In particular the biosensor system has great potential to be developed into a tool to measure early changes in neurodegenerative disease where early diagnostics are desperately needed. The development of treatments for neurodegenerative diseases including Huntington's (HD), Alzheimer's (AD) and Motor Neuron Disease (MND) remains one of the toughest scientific challenges of our times. Between 2002 and 2012 only one of $244 \mathrm{AD}$ clinical trials yielded a treatment with therapeutic benefit ${ }^{30}$. Similarly Motor Neuron disease remains largely untreatable with only two drugs approved by the US Food and Drugs Administration that mildly delay disease progression

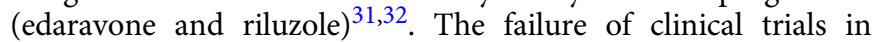
neurodegenerative diseases may arise from trials beginning too late in the disease course. In turn, beginning trials at presymptomatic stages of disease has been hampered by a lack of 
a

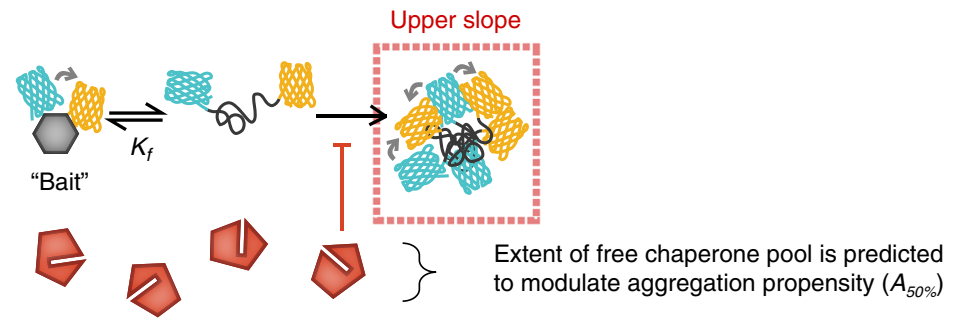

b

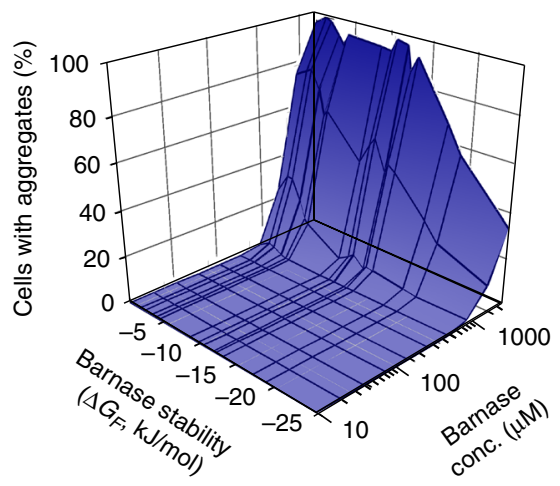

d

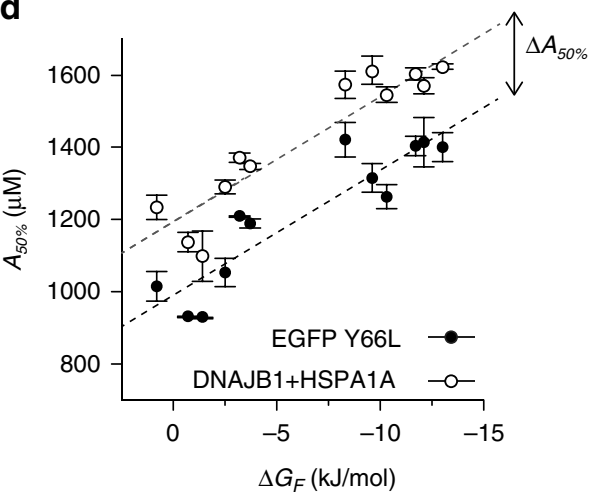

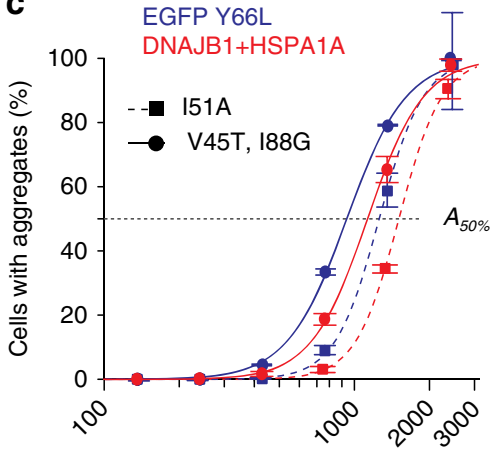

Barnase concentration $(\mu \mathrm{M})$

e

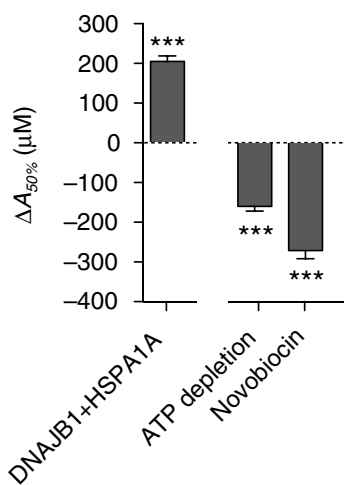

Fig. 5 Quantifying proteostasis by changes in biosensor aggregation propensity $\left(A_{50 \%}\right)$. a Conceptual framework for how chaperone levels affect barnasebiosensor aggregation. b Baseline barnase aggregation "landscape" in cells as measured by the proportion of cells in the Upper-slope flow cytometry population. Data show mean of three replicates for each barnase mutant coexpressed with Y66L EGFP control. c Impact on chaperone overexpression on aggregation landscape. Data shows two of the barnase mutants and corresponding treatment regimes. Data points are means \pm SD of three replicates. Chaperone treatment vs. control, $p<0.0001$, extra sum-of-squares $\mathrm{F}$ test. $\mathbf{d}$ Impact of chaperone overexpression on $A_{50 \%}$ values. Each data point reflects one mutant (means $\pm \mathrm{SEM}$ of three replicates). Lines show linear regressions with same slope (preferred model by extra sum of squares $\mathrm{F}$-test, $p=0.57)$. $\Delta A_{50 \%}$ is the translational offset, calculated for each mutant (example indicated by arrow). e Impact of proteostasis on changes in $A_{50 \%}\left(\Delta A_{50 \%}\right)$. Data show chaperone co-expression (compared to Y66L EGFP negative control), treatment with proteostasis-modulating drugs (compared to untreated control). Data are means \pm SEM of 12 mutants of barnase. Results of Wilcoxon signed rank test are shown: Results coded as ${ }^{\star \star *} p<0.001$

biomarkers of presymptomatic disease progression ${ }^{33}$. Hence approaches, such as ours described here, provide important foundation stones for tackling this great challenge.

\section{Methods}

Expression constructs. A toolkit for FRET biosensor comprising a combinatorial library of circularly permuted mTFP1 and Venus fluorescent proteins (cpFRET library in $\mathrm{pTriEx} 4$ expression vector) was used ${ }^{34}$. Barnase mutants were synthesized (Thermo Fisher Scientific) and inserted via PCR-mediated cloning with XmaI and NotI flanking restriction sites between BspEI (complementary to XmaI) and NotI sites of the cpFRET library such that barnase was fused to mTFP1 and Venus at the amino and carboxy termini, respectively. Additional single mutations were isolated from double-mutant constructs using BspEI and XbaI restriction sites. All constructs were verified by sequencing and the basic construct sequence is shown in Supplementary Table 3. Plasmids expressing HSPA1A, DNAJB1 and mCherry were prepared as described previously ${ }^{35,36}$. pCMV6-AC BAG1 (cat\# SC319483), pCMV6-AC HSF1 (cat\# SC321225) and pCMV6-Entry myc-HSP70AA1 (cat\# RC212496) were purchased from Origene.
Cell culture. AD293 and HEK293 cell-lines (from lab cultures orginally obtained from ATCC) were used in this study and tested and cleared for mycoplasma. Cells were not tested for cross-contamination of other cell lines or misidentification. AD293 cells were maintained in Dulbecco's modified Eagles Medium (DMEM) supplemented with $2 \mathrm{mM}$ L-glutamine, $200 \mathrm{U} \mathrm{mL}^{-1}$ penicillin/streptomycin and $10 \% \mathrm{v} / \mathrm{v}$ fetal bovine serum (Thermo Fisher Scientific) in a humidified $37^{\circ} \mathrm{C}$ incubator with $5 \% \mathrm{v} / \mathrm{v}$ atmospheric $\mathrm{CO}_{2}$. HEK293T cell-lines were maintained as for AD293 cells except DMEM was supplemented with $2 \mathrm{mM}$ L-alanyl-L-glutamine, $100 \mathrm{U} \mathrm{mL}^{-1}$ penicillin/streptomycin and $10 \% \mathrm{v} / \mathrm{v}$ fetal bovine serum. For biosensor screening, $2 \times 10^{5} \mathrm{AD} 293$ cells were seeded on poly-L-lysine coated 12well plates and transfected using $1.6 \mu \mathrm{g}$ DNA and $4 \mu \mathrm{L}$ Lipofectamine 2000 as per the manufacturer's directions. For urea denaturation, flow cytometry and microscopy, $5 \times 10^{4}$ HEK293T cells were seeded on poly-L-lysine coated 48 -well plates (Corning) or 8-well $\mu$-slides (for microscopy, Ibidi). Cells were transfected using $0.25 \mu \mathrm{g}$ DNA, $0.5 \mu \mathrm{L}$ P3000 reagent and $0.75 \mu \mathrm{L}$ Lipofectamine 3000 according to the manufacturer's instructions.

Drug treatments. To deplete ATP, cells were kept in glucose-free DMEM (Thermo Fisher Scientific) for $16 \mathrm{~h}$ then treated with $10 \mathrm{mM}$ 2-deoxy-d-glucose 


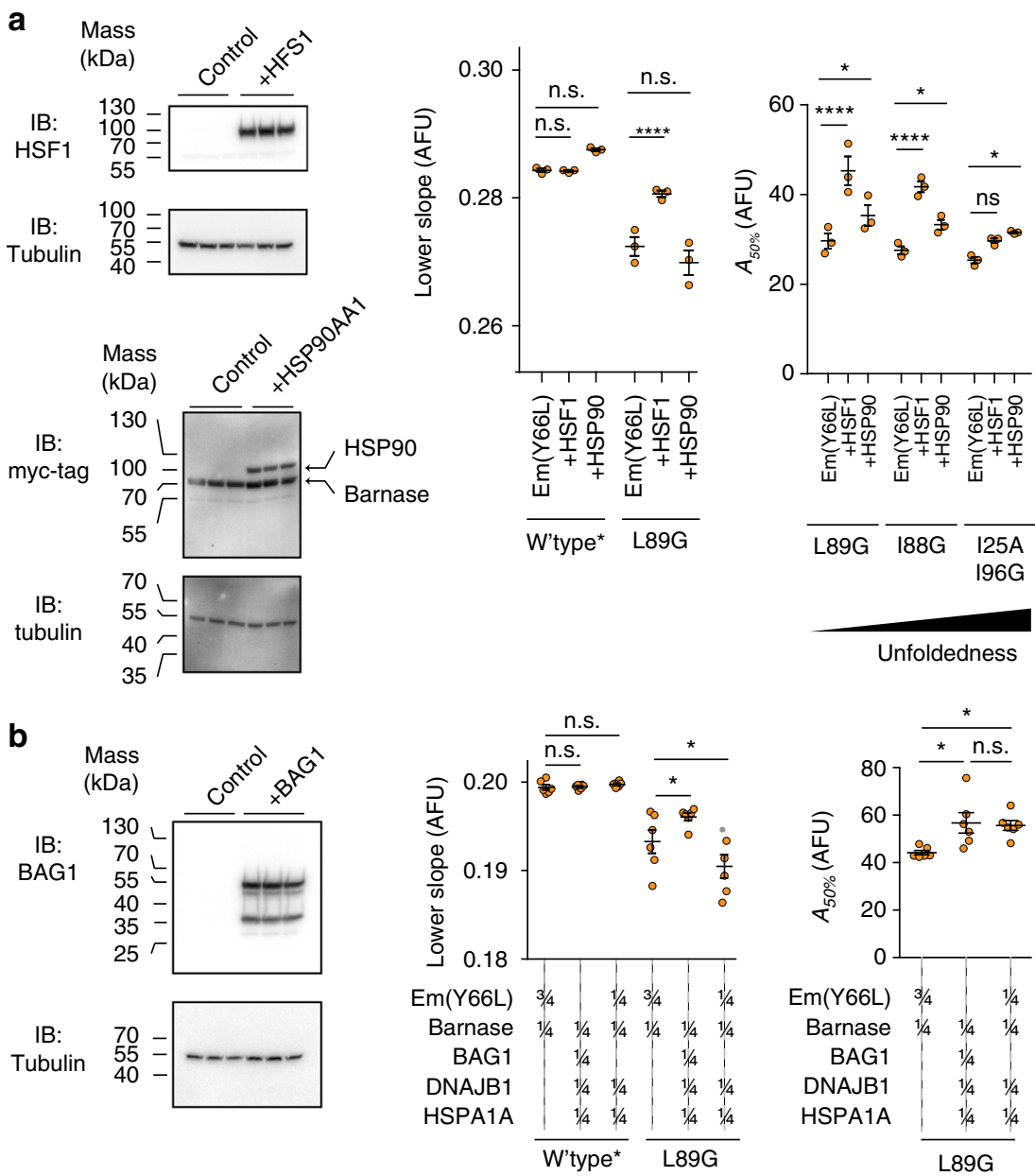

Fig. 6 Probing changes in proteostasis through selective activation of different chaperone systems. a Investigation of the effect of induction of the heat shock response by HSF1 overexpression or selective overexpression of HSP90. Shown on left are western blots of the HEK293T cells matched for total protein via a BCA assay. These cells were co-transfected with HSF1 (or myc-tagged HSP90 or control of (non-fluorescent) Y66L Emerald fluorescent protein) and the L89G barnase biosensor (which also contains a myc tag). The right graphs show the Lower-slope and $A_{50 \%}$ analyses of these treatments and one or three of the biosensor variants as indicated vs. the wild-type ${ }^{\star}$ variant. Bars indicate means $\pm \mathrm{SEM}$. The right panels were analysed via a two-way ANOVA subjected to a Dunnett's post-hoc test, results coded as ${ }^{\star \star \star \star} p<0.0001,{ }^{\star \star \star} p<0.001,{ }^{\star \star} p<0.01,{ }^{\star} p<0.05$, ns $=>0.05$. b Same paradigm as panel a. In this case, BAG1 was cotransfected with the barnase biosensor, HSP40 (DNAJB1) and HSP70 (HSPA1A). The right panels show the proportional dosage of each construct (by mass of DNA) in the transfection. Bars indicate means \pm SEM. The right panel Lower-slope graph was analysed via a two-way ANOVA subjected to Dunnett's post hoc test and the $A_{50 \%}$ graph was analysed via a one-way ANOVA subjected to a Tukey's post-hoc test. Results coded as ${ }^{\star * \star *} p<0.0001,{ }^{\star \star *} p<0.001,{ }^{* \star} p<0.01,{ }^{\star} p<0.05$, ns $=>0.05$

(Sigma) and $0.5 \mu \mathrm{M}$ valinomycin (Sigma) in glucose-free DMEM for $45 \mathrm{~min}$. ATP levels in ATP-depleted and control (untreated) cells were measured using the ATPlite luminescence assay system (Perkin Elmer) on a ClarioStar microplate reader, as per the manufacturer's protocol. HSP90 was inhibited with $800 \mu \mathrm{M}$ novobiocin in maintenance media for $6 \mathrm{~h}$. HSP70 was inhibited with $20 \mu \mathrm{M}$ VER155008 (Sigma cat \#SML0271) in maintenance media for $18 \mathrm{~h}$.

FRET biosensor screen. The cpFRET library containing wild type $(\mathrm{H} 102 \mathrm{~A})$ barnase was transfected into AD293 cells. $24 \mathrm{~h}$ post-transfection cells were washed with PBS then lysed by extrusion through a 27 gage syringe in native lysis buffer (20 mM Tris pH 8.0, $2 \mathrm{mM} \mathrm{MgCl}_{2}, 1 \% \mathrm{v} / \mathrm{v}$ Triton X-100, $1 \times$ EDTA-free protease inhibitor (Roche), $150 \mathrm{mM} \mathrm{NaCl}, 20 \mathrm{U} \mathrm{mL}^{-1}$ benzonase, $1 \mathrm{mM} \mathrm{PMSF}$ ). Lysate was centrifuged at $100,000 \times \mathrm{g}$ for $30 \mathrm{~min}$ at $4^{\circ} \mathrm{C}$ to remove cell debris. $80 \mu \mathrm{L}$ supernatant was added to $200 \mu \mathrm{L}$ native lysis buffer or native lysis buffer with $6 \mathrm{M}$ urea in a 96-well plate. mTFP1 was excited at $462 \mathrm{~nm}$ and emission spectra were collected from 480 to $600 \mathrm{~nm}$ using a Varioskan Flash microplate reader (Thermo Fisher Scientific). Relative FRET efficiency was calculated as $A /(D+A)$; that is, fluorescence intensity at the acceptor $(A)$ maximum $(532 \mathrm{~nm})$ divided by the sum of intensities at the donor $(D)(492 \mathrm{~nm})$ and acceptor maxima. Because the readings were ratiometric, we deemed it unnecessary to normalize protein concentration in the lysates.
Recombinant production of the Venus cp173 construct. The Venus cp173 construct in the pTriEx based vectors was expressed in T7 Express E. coli (NEB) using ampicillin as the selection antibiotic. $10 \mathrm{~mL}$ of an overnight starter culture (grown in $2 \times \mathrm{YT}$ at $37^{\circ} \mathrm{C}$ in a shaking incubator), was inoculated in $1 \mathrm{~L}$ of $2 \times \mathrm{YT}$ and grown to an $\mathrm{OD}_{600 \mathrm{~nm}}$ of $0.5 \mathrm{AU}$ at $37^{\circ} \mathrm{C}$ in a shaking incubator. The culture was cooled to $18{ }^{\circ} \mathrm{C}$ and then expressed was induced with $0.4 \mathrm{mM}$ Isopropyl $\beta-\mathrm{D}-1$ thiogalactopyranoside with cells grown overnight at $18^{\circ} \mathrm{C}$ in a shaking incubator. Cells were pelleted $\left(5000 \times g ; 4^{\circ} \mathrm{C}\right)$ and resuspended in $100 \mathrm{mM}$ Tris, pH 8.0 supplemented with Complete EDTA-free protease inhibitor cocktail (Roche) and 1 $\mathrm{mM}$ phenylmethylsulfonyl fluoride. Hen egg white lysozyme was added to a concentration of $1 \mathrm{mg} \mathrm{mL}^{-1}$ and the lysate was frozen at $-20^{\circ} \mathrm{C}$. The lysate was thawed, Benzonase nuclease was added according to the manufacturer's instructions (EMD-Millipore). The debris was pelleted and removed by centrifugation $\left(16,000 \times g ; 20 \mathrm{~min} ; 4^{\circ} \mathrm{C}\right)$. Imidazole was added to the supernatant to a concentration of $5 \mathrm{mM}$ and the solution was applied to a $1 \mathrm{~mL}$ His-tag column preequilibrated in binding buffer (PBS and $5 \mathrm{mM}$ imidazole). The column was washed with $20 \mathrm{~mL}$ column buffer and then with PBS and $25 \mathrm{mM}$ imidazole until no proteins further eluted (as assessed by Bradford assay). The column was then washed with $10 \mathrm{~mL}$ PBS and $50 \mathrm{mM}$ imidazole before elution with PBS and 200 $\mathrm{mM}$ imidazole. Eluted proteins from the most concentrated fractions were buffer exchanged into PBS using a PD-10 column (GE Healthcare). Proteins were immediately snap frozen in liquid nitrogen and stored at $-80^{\circ} \mathrm{C}$ until further use. 
Recombinant production of the barnase biosensors. The barnase constructs in the pTriEx based vectors were expressed in T7 Express E. coli (NEB) using ampicillin as the selection antibiotic. $10 \mathrm{~mL}$ of an overnight starter culture (grown in $2 \times \mathrm{YT}$ at $37^{\circ} \mathrm{C}$ in a shaking incubator), was inoculated in $100 \mathrm{~mL}$ of $2 \times \mathrm{YT}$ and grown to an $\mathrm{OD}_{600 \mathrm{~nm}}$ of $0.5 \mathrm{AU}$ at $37^{\circ} \mathrm{C}$ in a shaking incubator. The culture was cooled to $18{ }^{\circ} \mathrm{C}$ and expression was induced with $0.4 \mathrm{mM}$ Isopropyl $\beta$-D-1thiogalactopyranoside with cells grown overnight at $18^{\circ} \mathrm{C}$ in a shaking incubator. Cells were pelleted $\left(5000 \times g ; 4^{\circ} \mathrm{C}\right)$ and resuspended in phosphate buffered saline (PBS) supplemented with Complete EDTA-free protease inhibitor cocktail (Roche). Hen egg white lysozyme was added to a concentration of $1 \mathrm{mg} \mathrm{mL}^{-1}$ and the lysate was frozen at $-20^{\circ} \mathrm{C}$. The lysate was thawed, Benzonase nuclease was added according to the manufacturer's instructions (EMD-Millipore). The debris was pelleted and removed by centrifugation $\left(16,000 \times g ; 15 \mathrm{~min} ; 4^{\circ} \mathrm{C}\right)$. Imidazole was added to the supernatant to a concentration of $20 \mathrm{mM}$ and the solution was applied to a His SpinTrap column (GE Life Sciences) pre-equilibrated in binding buffer (PBS and $20 \mathrm{mM}$ imidazole). Proteins were purified as per the manufacturer's directions, using PBS and $200 \mathrm{mM}$ imidazole as the elution buffer. Proteins were used immediately.

Urea denaturation stability measurements. 384-well plates were prepared with $80 \mu \mathrm{L}$ of a concentration series of urea $(0 \mathrm{M}$ to $\sim 6 \mathrm{M})$ in phosphate buffered saline (PBS). For the recombinant purified proteins, $5 \mu \mathrm{L}$ of protein was used in the assay. For the curves performed with cell lysates, cells were lysed $24 \mathrm{~h}$ after transfection by pipetting in native lysis buffer. Aggregates and cell debris were pelleted by centrifugation at $16,000 \times g$ for $10 \mathrm{~min}$ at $4{ }^{\circ} \mathrm{C}$. $5 \mu \mathrm{L}$ supernatant was added to each urea concentration. Samples were not matched for protein concentration; however, as the measurements were ratiometric and both fluorophores were on the same molecule, concentration was not expected to influence results. Fluorescence readings ( $430 \mathrm{~nm}$ excitation, $492 \mathrm{~nm}$ emission and 532 emission) were measured at $23^{\circ}$ $\mathrm{C}$ using a ClarioStar microplate reader every $15 \mathrm{~min}$ for $4 \mathrm{~h}$. Readings were stable for the duration of the experiment. Relative FRET efficiencies (calculated as $A /(D$ $+A$ ) as per the FRET biosensor screen) were averaged across readings and fit to a two-state unfolding model with terms for pre- and post-transition baselines (Equation 2).

$$
\mathrm{FRET}=\frac{\left(\alpha_{N}+\beta_{N} \cdot[U]\right)+\left(\alpha_{D}+\beta_{D} \cdot[U]\right) \cdot \mathrm{e}^{\frac{-m}{R T}\left(D_{50 \%}-[U]\right)}}{1+\mathrm{e}^{-\frac{m}{R T}\left(D_{50 \%}-[U]\right)}}
$$

Where $[U]$ is the urea concentration, $D_{50 \%}$ is the urea concentration at which barnase is $50 \%$ denatured, $m$ is the cooperativity value and $m\left(D_{50 \%}-[U]\right)=\Delta G_{F}$, and $\alpha$ and $\beta$ describe the FRET baselines of barnase in the native $\left(\alpha_{N}, \beta_{N}\right)$ and denatured $\left(\alpha_{D}, \beta_{D}\right)$ conformations. That is, $\alpha$ is the FRET signal of barnase in the native $\left(\alpha_{N}\right)$ or denatured $\left(\alpha_{D}\right)$ conformation when $[U]=0$ and $\beta$ is the rate of change of the FRET signal with increasing $[U]$. As small error in the cooperativity value $m$ has large impact on $\Delta G_{F}, m$ was constrained as a shared parameter for all mutants. To enable fits for destabilized mutants that are partially unfolded at $0 \mathrm{M}$ urea, pre- and post-transition baselines were shared for all mutants. Reported $\Delta G_{F}$ were averages of three experiments fit independently.

Microscopy. To estimate cell volume, cells expressing Venus (cp173) were imaged $24 \mathrm{~h}$ after transfection on a Leica TCS SP5 Confocal microscope with a HCX APO CS $63 \times 1.40$ Oil objective and 1 Airy pinhole as Z-stacks in $0.21 \mu \mathrm{m}$ steps $(514 \mathrm{~nm}$ excitation, 520-650 nm emission) and analyzed using ImageJ. To measure FRET in intact cells, cells were imaged with a Leica SP5 confocal microscope using a HCX APO CS $63 \times 1.40$ Oil objective and 1 Airy pinhole. Emission spectral scans were acquired with a $405 \mathrm{~nm}$ excitation laser, from 480 to $576.6 \mathrm{~nm}$ in $3.45 \mathrm{~nm}$ steps with a collection bandwidth window of $10 \mathrm{~nm}$.

Immunoprecipitation for western blotting. In total $1.25 \times 10^{5}$ HEK293T cells were seeded into $25 \mathrm{~cm}^{2}$ flasks and transfected the following day with $6.25 \mu \mathrm{g}$ DNA constructs, $12.5 \mu \mathrm{L} \mathrm{P} 3000$ and $18.75 \mu \mathrm{L}$ Lipofectamine 3000 (Life Technologies) according to the manufacturer's instructions (Life Technologies). Cells were harvested at $24 \mathrm{~h}$ post-transfection for drug treatments and $48 \mathrm{~h}$ for co-expressions. Cells were harvested by gently pipetting with PBS. Cells were pelleted by centrifugation at $120 \times g$ for $6 \mathrm{~min}$ and resuspended in lysis buffer $(0.5 \% \mathrm{v} / \mathrm{v}$ IGEPAL, $50 \mathrm{mM}$ Tris, $5 \mathrm{mM} \mathrm{MgCl}$, $\mathrm{pH} 7.4$ supplemented with EDTA-free protease inhibitor (Roche). Lysate was incubated on ice for $10 \mathrm{~min}$. Debris was removed by centrifugation $\left(13,000 \mathrm{rpm} ; 10 \mathrm{~min} ; 4^{\circ} \mathrm{C}\right)$ and the supernatant placed in a new microcentrifuge tube. For samples matched for barnase fluorescence, fluorescence was measured on a ClarioStar microplate reader $(e x=513 \pm 10 \mathrm{~nm}$, $\mathrm{em}=530 \pm 30 \mathrm{~nm}$ ). $20 \mu \mathrm{L}$ of GFP-Trap_MA (Chromotek) beads prewashed in wash buffer (0.5\% v/v IGEPAL, $50 \mathrm{mM}$ Tris, $150 \mathrm{mM} \mathrm{NaCl}, 5 \mathrm{mM}$ EDTA, $\mathrm{pH}$ 7.4) was added to each sample and the mixture was incubated for $2 \mathrm{~h}$ at $4{ }^{\circ} \mathrm{C}$ with constant rotation. The unbound fraction was removed from beads immobilized with a magnet, and beads were washed three times with wash buffer at room temperature. Proteins were eluted off the beads by boiling the beads in SDS-PAGE Laemmli sample buffer for $10 \mathrm{~min}$.
Western blotting. Proteins were transferred to PVDF membrane, blocked in blocking buffer (5\% w/v skim milk powder in PBS-T) for $1 \mathrm{~h}$ at room temperature and then incubated with primary antibody in blocking buffer. The following antibodies were incubated overnight at $4{ }^{\circ} \mathrm{C}$ : anti-HSPA1A (Origene, cat \#TA500772, 1:10,000), anti-HSF1 (Abcam, cat \#ab52757, 1:40,000), anti-BAG1 (Abcam, cat \#ab32109, 1:750), and anti-myc (Thermofisher, cat \#13-2500, 1:1,000). The blots were washed in PBS-T and then incubated with either anti-rabbit secondary antibody (Invitrogen, cat \#65-6120, 1:20,000) or anti-mouse secondary antibody (Invitrogen, cat $\# 31430,1: 20,000$ ) in PBS-T for $1 \mathrm{~h}$ at room temperature. Proteins were detected by an enhanced chemiluminescence kit (Clarity, BioRad). The uncropped blots are shown in Supplementary Fig. 7.

Sample preparation for proteomics. In total $3.5 \times 10^{6}$ HEK293T cells were seeded into $75 \mathrm{~cm}^{2}$ flasks and transfected the following day with either WT* or I25A, I96G barnase constructs ( $18.75 \mu \mathrm{g}$ DNA, $37.5 \mu \mathrm{L}$ P3000 and $56.25 \mu \mathrm{L}$ Lipofectamine 3000 (Life Technologies)) according to the manufacturer's instructions (Life Technologies). The experiment was designed as 4 biological matched pair replicates. Media was refreshed $5 \mathrm{~h}$ after transfection. At $24 \mathrm{~h}$ post-transfection, cells were gently rinsed with PBS and harvested in PBS by gently pipetting. Cells were pelleted $(120 \times g$; $6 \mathrm{~min}$; room temperature $)$ and resuspended in $1 \mathrm{~mL}$ PBS and pelleted again $(4000 \times g ; 6 \mathrm{~min}$; room temperature). The pellet was resuspended in ice-cold $200 \mu \mathrm{L}$ HENG buffer $(50 \mathrm{mM}$ HEPES-KOH, $\mathrm{pH} 7.9,150 \mathrm{mM} \mathrm{NaCl}, 20 \mathrm{mM}$ $\mathrm{Na}_{2} \mathrm{MoO}_{4}, 2 \mathrm{mM}$ EDTA, 5\% v/v glycerol, 1 mM PMSF and Complete EDTA-free protease inhibitor (Roche)). Cells were mechanically lysed using a cryomill (Precellys 24; Bertin) after addition of $40 \mu \mathrm{L} 0.15 \mathrm{~mm}$ zirconium oxide beads using $3 \times$ ( $30 \mathrm{~s}$ on, $30 \mathrm{~s}$ off) cycles at $6800 \mathrm{rpm}$ with temperature monitored and maintained at $10^{\circ} \mathrm{C}$ or less. The resultant lysate was supplemented to $500 \mu \mathrm{L}$ with HENG buffer and pelleted $\left(16,000 \times g ; 10 \mathrm{~min} ; 4^{\circ} \mathrm{C}\right)$. The supernatant was removed and matched for Venus fluorescence $(\mathrm{ex}=514 \mathrm{~nm}, \mathrm{em}=527 \mathrm{~nm})$ using a platereader (ClarioSTAR; BMG). $350 \mu \mathrm{L}$ of lysate was added to $30 \mu \mathrm{L}$ GFP-Trap agarose beads (Chromatek) pre-washed and equilibrated in HENG buffer. The solution was incubated for $2 \mathrm{~h}$ at $4{ }^{\circ} \mathrm{C}$ with constant rotation. Beads were collected by pelleting $\left(2000 \times g ; 2 \mathrm{~min} ; 4^{\circ} \mathrm{C}\right)$ and washed twice with HENG buffer by pelleting and resuspension. Beads were then washed twice more with $1 \mathrm{mM}$ triethylammonium bicarbonate (TEAB) buffer. Proteins were eluted by addition of $30 \mu \mathrm{L} 0.1 \% \mathrm{v} / \mathrm{v}$ formic acid, $5 \% \mathrm{v} / \mathrm{v}$ trifluoroethanol, $1 \mathrm{mM}$ tris(2-carboxyethyl)phosphine for 5 min at room temperature. The supernatant was collected after pelleting $(2000 \mathrm{~g} ; 2$ min; room temperature) and adjusted to a final concentration of $100 \mathrm{mM}$ TEAB by addition of $1 \mathrm{M}$ stock solution (and the $\mathrm{pH}$ was validated to be about 7 after this treatment). Proteins were reduced using $10 \mathrm{mM}$ tris (2-carboxyethyl)phosphine, $\mathrm{pH} 8.0$, and alkylated with $10 \mathrm{mM}$ iodoacetamide for $45 \mathrm{~min}$ and then digested by addition of $0.25 \mu \mathrm{g}$ trypsin and incubation overnight at $37^{\circ} \mathrm{C}$. Peptides (in a volume of $50 \mu \mathrm{L}$ ) were differentially labelled by reductive dimethyl labelling using $2 \mu \mathrm{L}$ of $4 \%$ (vol/vol) formaldehyde $-\mathrm{CH}_{2} \mathrm{O}$ (light label), $\mathrm{CD}_{2} \mathrm{O}$ (medium label), ${ }^{13} \mathrm{CD}_{2} \mathrm{O}$ (heavy) (mixed design across replicates) and $2 \mu \mathrm{L}$ of $0.6 \mathrm{M}$ sodium cyanoborohydride for $1 \mathrm{~h}$ at room temperature. The reaction was quenched by addition of $8 \mu \mathrm{L}$ of $1 \%$ ammonium hydroxide followed by $8 \mu \mathrm{L}$ of neat formaldehyde.

NanoESI-LC-MS/MS analysis. Samples were analysed by nanoESI-LC-MS/MS using a Q Exactive Plus mass spectrometer (Thermo Scientific, San Jose, CA) fitted with a nanoflow reversed-phase-HPLC (Ultimate 3000 RSLC, Dionex). The nanoLC system was equipped with an Acclaim Pepmap nano-trap column (Dionex $-\mathrm{C} 18,100 \AA, 75 \mu \mathrm{m} \times 2 \mathrm{~cm}$ ) and an Acclaim Pepmap RSLC analytical column (Dionex-C18, $100 \AA, 75 \mu \mathrm{m} \times 50 \mathrm{~cm}$ ). Typically for each LC-MS/MS experiment, 5 $\mu \mathrm{L}$ of the peptide mix was loaded onto the enrichment (trap) column at an isocratic flow of $5 \mu \mathrm{L} \mathrm{min}^{-1}$ of $3 \% \mathrm{CH}_{3} \mathrm{CN}$ containing $0.1 \%$ formic acid for $5 \mathrm{~min}$ before the enrichment column was switched in-line with the analytical column. The eluents used for the LC were $0.1 \% \mathrm{v} / \mathrm{v}$ formic acid (solvent A) and $100 \% \mathrm{CH}_{3} \mathrm{CN} / 0.1 \%$ formic acid $\mathrm{v} / \mathrm{v}$ (solvent $\mathrm{B}$ ). The gradient used $\left(300 \mathrm{~nL} \mathrm{~min}{ }^{-1}\right)$ was from $3 \mathrm{~B}$ to $20 \%$ B for $35 \mathrm{~min}, 20 \mathrm{~B}$ to $45 \% \mathrm{~B}$ in $8 \mathrm{~min}, 45 \mathrm{~B}$ to $80 \% \mathrm{~B}$ in $2 \mathrm{~min}$ and maintained at $80 \% \mathrm{~B}$ for the final $3 \mathrm{~min}$ before equilibration for $6 \mathrm{~min}$ at $3 \% \mathrm{~B}$ prior to the next analysis. All spectra were acquired in positive mode with full scan MS spectra scanning from $\mathrm{m} / \mathrm{z} 375-1400$ in the FT mode at 70,000 resolution after accumulating to a target value of $3.00 \mathrm{e}^{6}$ with maximum accumulation of $50 \mathrm{~ms}$. Lockmass of 445.120024 was used. Data dependant HCD MS/MS of the 15 most intense peptide ions with charge states $>1$ was performed, using an isolation width of 1.2, a target value of $1.00 \mathrm{e}^{5}$, a maximum accumulation time of $120 \mathrm{~ms}$, a normalized collision energy of $30 \%$, and a 35,000 mass resolving power. Dynamic exclusion was used for $30 \mathrm{~s}$.

Data analysis was carried out using Proteome Discoverer (version 2.1.0.81; Thermo Scientific) with the Mascot search engine (Matrix Science version 2.4.1). Data were filtered against the Swissprot Homo sapiens database (version 2015_07: Jun-24, 2015; 548872 entries). The search was conducted with 20 ppm MS tolerance, $0.8 \mathrm{Da} \mathrm{MS} / \mathrm{MS}$ tolerance, 2 missed cleavages allowed. The following modifications were allowed: Oxidation (M), Acetylation (Protein N-term), Dimethylation (K), Dimethylation (N-Term), ${ }^{2} \mathrm{H}(4)$ Dimethylation: (K), ${ }^{2} \mathrm{H}(4)$ Dimethylation (N-term), ${ }^{2} \mathrm{H}(6){ }^{13} \mathrm{C}(2)$ Dimethylation (K), ${ }^{2} \mathrm{H}(6){ }^{13} \mathrm{C}(2)$ Dimethylation (N-term) (Variable); Carbamidomethyl (C) (Fixed). The false discovery rate (FDR) maximum was set to $0.1 \%$ at the peptide identification level 
(actual was $0.06 \%$ for each replicate) and $1 \%$ at the protein identification level. Proteins were filtered for those containing at least one unique peptide in all fou replicates. The common contaminant, Keratin, was excluded from the dataset. Peptide quantitation was performed in Proteome Discoverer v.2.1.0.81 using the precursor ion quantifier node. Dimethyl labelled peptide pairs (between two comparison of light, medium or heavy) were established with a $2 \mathrm{ppm}$ mass precision, a signal to noise threshold of 3 , and filtered for a minimum Mascot ion score of 30. The retention time tolerance of isotope pattern multiplets was set to $0.8 \mathrm{~min}$. Three single peak or missing channels were allowed for peptide identification. The protein abundance in each replicate was calculated by summation of the unique peptide abundances that were used for quantitation (light, medium and-or heavy dimethyl derivatives). In the cases where quan values were missing or were apparent outliers to the other two replicates, raw data was manually checked and added or adjusted where relevant. The protein ratios (from two of the light, medium and heavy labels) were manually calculated from the protein abundances as described previously ${ }^{37}$. Data were normalized to the Venus protein peptide abundances in the datasets. These correction values were multipliers of (replicate 1: 3.583), (replicate 2: 3.45), (replicate 3: 4.284) and (replicate 4: 5.027). Proteins were excluded that had an SD greater than $60 \%$. Student's t-test was performed to calculate the statistical significance.

Flow cytometry. After $24 \mathrm{~h}$ (drug treatments) or $48 \mathrm{~h}$ (cotransfections) of posttransfection, cells were washed once in PBS then harvested by gentle pipetting in PBS and transferred to a 96-well U-bottom microplate. $100 \mu \mathrm{L}$ cell suspension was analysed at $3 \mu \mathrm{L} \mathrm{s}^{-1}$ using the high throughput sampler in an LSRFortessa flow cytometer equipped with 405, 488, and $561 \mathrm{~nm}$ lasers (BD Biosciences). Forward scatter threshold was set to 5000. Acceptor (Venus) fluorescence was collected with the $488 \mathrm{~nm}$ laser and FITC (530/30) filter. Acceptor sensitized emission (FRET) and donor (mTFP1) fluorescence were collected with the $405 \mathrm{~nm}$ laser with PE (575/25) and V500 (525/50) filters, respectively. All flow cytometry data were processed with FlowJo (Tree Star Inc) to exclude cell debris, cell aggregates and untransfected cells.The Venus channel was compensated to remove bleedthrough from mTFP1 and FRET channels. mTFP1, FRET and Venus data were exported as csv files and analyzed in MATLAB (see section on data analysis). The gating strategy is explained in the associated Protocols Exchange manuscript ${ }^{38}$.

Cells were sorted using a Becton Dickinson FACSAria III sorter at the University of Melbourne Brain Centre flow cytometry facility. FRET and mTFP1 fluorescence were collected with the $405 \mathrm{~nm}$ laser with 582/15 and 510/50 filters, respectively. Cells were fixed in $2 \% \mathrm{v} / \mathrm{v}$ paraformaldehyde immediately after sorting and imaged on cover slips using Venus fluorescence as described above (without Zstacks).

Data analysis. Flow cytometry csv files were analyzed in MATLAB (MathWorks) to automatically classify cells as diffuse, or aggregated. The script is available upon request. The rational of the analysis is described here. The Lower-slope and Upperslope populations can be visually distinguished on a plot of the FRET channel against the donor channel. However, since the Lower-slope gradient differed for each barnase mutant, we could not use of a single Upper-slope gate for aggregate classification. Our approach was to fit the slope of the Lower-slope population, then classify any cells with substantially higher FRET than this belonging to the Upper-slope population. Since many datasets contained high numbers of cells with barnase aggregation, fitting methods designed to ignore outliers were insufficient to exclude high-FRET cells from the fit. To overcome this we (i) implemented a preprocessing step in FlowJo to immediately exclude very high-FRET cells (i.e., higher FRET than all wild-type ${ }^{*}$ barnase cells) from the fit, then (ii) performed a robust fit multiple times, excluding data that deviated significantly above the slope after each iteration. To determine significant deviation above the slope, we used the standard deviation of cells expressing wild-type* barnase (which contains no barnase aggregates) from the slope as an estimate of the expected standard deviation of diffuse cells around the slope for all mutants. We excluded cells that were greater than 2 standard deviations above the slope from the subsequent fitting iteration. We found that four iterations was sufficient to fit the Lower-slope population satisfactorily.

For analysis of the Lower-slope population the Venus acceptor fluorescence was restricted to a range up to 0.2 of the maximum dynamic range (which corresponded to typically $560-4600 \mathrm{AFU}$ on the BD LSRFortessa flow cytometer). This concentration range provided a trade-off between having low barnase concentration at which the model predicts chaperone engagement will be most pronounced, having enough cells for high quality data and having sufficient signal above background to accurately determine the Lower-slope gradient.

For analysis of $A_{50 \%}$, cells were binned into expression levels using Venus fluorescence, with 9 logarithmic bins evenly spanning the lower and upper bounds of fluorescence (e.g., $10^{2.5}, 10^{2.75}, 10^{3}, 10^{3.25}, 10^{3.5}, 10^{3.75}, 10^{4}, 10^{4.25}$, and $10^{4.5}$ $\mathrm{AFU}$ ). The percent of cells with aggregates (i.e., the Upper-slope population) were calculated for each expression bin. For each dataset, a Boltzmann sigmoidal curve was fit to the relationship between percent of cells with aggregates and $\log _{10}$ (expression bin midpoint) using Graphpad Prism. For the most stable mutants there was very little aggregation, even at high expression level, resulting in large uncertainty in the fits. Therefore, data from wild-type*, V45T, and I55G were excluded from the analysis. The expression level at which there was $50 \%$ aggregation $\left(A_{50 \%}\right)$ was determined from the sigmoidal fits. For hypothesis testing (i.e., to determine whether a treatment resulted in a significant change in aggregation propensity) the difference between average $A_{50 \%}$ of control and treatment was calculated for each barnase mutant (excluding wild-type*, V45T, and $\mathrm{I} 55 \mathrm{G})$. A Wilcoxon signed rank test was used to calculate whether the difference was significantly different to zero, treating the twelve mutants as replicate values.

Equation 1 was derived as described in Supplementary Note 2 and applied to our data as described in Supplementary Note 3.

Statistics. All statistics (other than the Proteomics) were performed in Prism software version 5 (GraphPad). The statistical tests and results are described in the figure legends.

Data availability. Raw data not presented in the manuscript are available upon request.

Received: 6 August 2017 Accepted: 11 December 2017 Published online: 18 January 2018

\section{References}

1. Hartl, F. U., Bracher, A. \& Hayer-Hartl, M. Molecular chaperones in protein folding and proteostasis. Nature 475, 324-332 (2011).

2. Schneider, K. \& Bertolotti, A. Surviving protein quality control catastrophes from cells to organisms. J. Cell Sci. 128, 3861-3869 (2015).

3. Gidalevitz, T., Ben-Zvi, A., Ho, K. H., Brignull, H. R. \& Morimoto, R. I. Progressive disruption of cellular protein folding in models of polyglutamine diseases. Science 311, 1471-1474 (2006).

4. Gupta, R. et al. Firefly luciferase mutants as sensors of proteome stress. Nat. Methods 8, 879-U155 (2011)

5. Liu, Y., Zhang, X., Chen, W., Tan, Y. L. \& Kelly, J. W. Fluorescence turn-on folding sensor to monitor proteome stress in live cells. J. Am. Chem. Soc. 137, 11303-11311 (2015).

6. Winkler, J. et al. Quantitative and spatio-temporal features of protein aggregation in Escherichia coli and consequences on protein quality control and cellular ageing. Embo. J. 29, 910-923 (2010).

7. Ebbinghaus, S., Dhar, A., McDonald, D. J. \& Gruebele, M. Protein folding stability and dynamics imaged in a living cell. Nat. Methods 7, 319-323 (2010).

8. Matouschek, A., Serrano, L., Meiering, E. M., Bycroft, M. \& Fersht, A. R. The folding of an enzyme .5. H/H-2 exchange nuclear-magnetic-resonance studies on the folding pathway of barnase-complementarity to and agreement with protein engineering studies. J. Mol. Biol. 224, 837-845 (1992).

9. Fersht, A. R. Protein folding and stability: the pathway of folding of barnase. FEBS Lett. 325, 5-16 (1993).

10. Serrano, L. et al. The folding of an enzyme. II. Substructure of barnase and the contribution of different interactions to protein stability. J. Mol. Biol. 224, 783-804 (1992).

11. Vu, N. D., Feng, H. \& Bai, Y. The folding pathway of barnase: the rate-limiting transition state and a hidden intermediate under native conditions. Biochemistry 43, 3346-3356 (2004)

12. Ishihama, Y. et al. Exponentially modified protein abundance index (emPAI) for estimation of absolute protein amount in proteomics by the number of sequenced peptides per protein. Mol. Cell. Proteom. 4, 1265-1272 (2005).

13. Palleros, D. R., Shi, L., Reid, K. L. \& Fink, A. L. hsp70-protein complexes. Complex stability and conformation of bound substrate protein. J. Biol. Chem. 269, 13107-13114 (1994).

14. Bukau, B. \& Horwich, A. L. The Hsp70 and Hsp60 chaperone machines. Cell 92, 351-366 (1998).

15. Matouschek, A. et al. Transient folding intermediates characterized by protein engineering. Nature 346, 440-445 (1990)

16. Ingolia, N. T., Lareau, L. F. \& Weissman, J. S. Ribosome profiling of mouse embryonic stem cells reveals the complexity and dynamics of mammalian proteomes. Cell 147, 789-802 (2011)

17. Princiotta, M. F. et al. Quantitating protein synthesis, degradation, and endogenous antigen processing. Immunity 18, 343-354 (2003).

18. Marcu, M. G., Schulte, T. W. \& Neckers, L. Novobiocin and related coumarin and depletion of heat shock protein 90 -dependent signaling proteins. J. Natl. Cancer Inst. 92, 242-248 (2000).

19. Conde, R., Belak, Z. R., Nair, M., O'Carroll, R. F. \& Ovsenek, N. Modulation of Hsf1 activity by novobiocin and geldanamycin. Biochem. Cell Biol. 87, 845-851 (2009).

20. Moore, C. \& Pressman, B. C. Mechanism of action of valinomycin on mitochondria. Biochem. Biophys. Res. Commun. 16, 562 (1964). 
21. McComb, R. B. \& Yushok, W. D. Metabolism of ascites tumor cells. Iv. Enzymatic reactions involved in adenosinetriphosphate degradation induced by 2-deoxyglucose. Cancer Res. 24, 198-205 (1964).

22. Williamson, D. S. et al. Novel adenosine-derived inhibitors of $70 \mathrm{kDa}$ heat shock protein, discovered through structure-based design. J. Med. Chem. 52, 1510-1513 (2009).

23. Schlecht, R. et al. Functional analysis of Hsp70 inhibitors. PLoS ONE 8, e78443 (2013).

24. Sondermann, H. et al. Structure of a Bag/Hsc70 complex: convergent functional evolution of Hsp70 nucleotide exchange factors. Science 291, 1553-1557 (2001).

25. Harrison, C. J., Hayer-Hartl, M., Di Liberto, M., Hartl, F. \& Kuriyan, J. Crystal structure of the nucleotide exchange factor GrpE bound to the ATPase domain of the molecular chaperone DnaK. Science 276, 431-435 (1997).

26. Liberek, K., Marszalek, J., Ang, D., Georgopoulos, C. \& Zylicz, M. Escherichia coli DnaJ and GrpE heat shock proteins jointly stimulate ATPase activity of DnaK. Proc. Natl Acad. Sci. USA 88, 2874-2878 (1991).

27. Hageman, J., van Waarde, M. A., Zylicz, A., Walerych, D. \& Kampinga, H. H. The diverse members of the mammalian HSP70 machine show distinct chaperone-like activities. Biochem. J. 435, 127-142 (2011).

28. Rampelt, H. et al. Metazoan Hsp70 machines use Hsp110 to power protein disaggregation. EMBO J. 31, 4221-4235 (2012).

29. Hohfeld, J., Cyr, D. M. \& Patterson, C. From the cradle to the grave: molecular chaperones that may choose between folding and degradation. EMBO Rep. 2, 885-890 (2001)

30. Cummings, J. L., Morstorf, T. \& Zhong, K. Alzheimer's disease drugdevelopment pipeline: few candidates, frequent failures. Alzheimers Res. Ther. 6 37 (2014)

31. Mitsumoto, H., Brooks, B. R. \& Silani, V. Clinical trials in amyotrophic lateral sclerosis: why so many negative trials and how can trials be improved? Lancet Neurol. 13, 1127-1138 (2014).

32. Writing Group, Edaravone ALS 19 Study Group. Safety and efficacy of edaravone in well defined patients with amyotrophic lateral sclerosis: a randomised, double-blind, placebo-controlled trial. Lancet Neurol. 16, 505-512 (2017).

33. Noelker, C., Hampel, H. \& Dodel, R. Blood-based protein biomarkers for diagnosis and classification of neurodegenerative diseases: current progress and clinical potential. Mol. Diagn. Ther. 15, 83-102 (2011).

34. Fritz, R. D. et al. A versatile toolkit to produce sensitive FRET biosensors to visualize signaling in time and space. Sci. Signal. 6, rs12 (2013).

35. Ormsby, A. R., Ramdzan, Y. M., Mok, Y. F., Jovanoski, K. D. \& Hatters, D. M. A platform to view huntingtin exon 1 aggregation flux in the cell reveals divergent influences from chaperones hsp40 and hsp70. J. Biol. Chem. 288, 37192-37203 (2013)

36. Polling, S. et al. Polyalanine expansions drive a shift into alpha-helical clusters without amyloid-fibril formation. Nat. Struct. Mol. Biol. 22, 1008-1015 (2015).

37. Kim, W. et al. Systematic and quantitative assessment of the ubiquitin-modified proteome. Mol. Cell 44, 325-340 (2011).
38. Wood, R. et al. Measuring proteostasis capacity using transiently transfected bait proteins by flow cytometry. Protocol Exchange https://doi.org/10.1038/ protex.2017.156 (2018)

\section{Acknowledgements}

This work was funded by grants from the Australian Research Council Future Fellowships scheme FT120100039 (D.M.H.), and National Health and Medical Research Council Project grants APP1049458 (D.M.H.) and APP1049459 (D.M.H.), and Human Frontier Science Program research grant RGP0022/2017 (D.M.H, S.E. and A.D.). S.E. acknowledges funding from the Cluster of Excellence RESOLV (EXC 1069) funded by the German Research Foundation (D.F.G.).

\section{Author contributions}

R.J.W. designed and performed the experiments and analytical approaches, and co-wrote the manuscript. A.R.O., M.R., D.C., G.E.R., A.S., T.V., S.E. and M.O. assisted in project design and/or performing experiments. A.D. developed the mathematical models. D.M. $\mathrm{H}$. devised the original concept of the project, oversaw its implementation and co-wrote the manuscript.

\section{Additional information}

Supplementary Information accompanies this paper at https://doi.org/10.1038/s41467 017-02562-5.

Competing interests: The authors declare no competing financial interests.

Reprints and permission information is available online at http://npg.nature.com/ reprintsandpermissions/

Publisher's note: Springer Nature remains neutral with regard to jurisdictional claims in published maps and institutional affiliations.

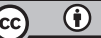

Open Access This article is licensed under a Creative Commons Attribution 4.0 International License, which permits use, sharing, adaptation, distribution and reproduction in any medium or format, as long as you give appropriate credit to the original author(s) and the source, provide a link to the Creative Commons license, and indicate if changes were made. The images or other third party material in this article are included in the article's Creative Commons license, unless indicated otherwise in a credit line to the material. If material is not included in the article's Creative Commons license and your intended use is not permitted by statutory regulation or exceeds the permitted use, you will need to obtain permission directly from the copyright holder. To view a copy of this license, visit http://creativecommons.org/ licenses/by/4.0/

(C) The Author(s) 2018 